Recepción: 15 / 04 / 2017

Aceptación: 01 / 05 / 2017

Ciencias Pedagógicas

Publicación: 15 / 05 / 2017

Revisión de Literatura

\title{
Interaprendizaje practico basado en métodos y competencias para optimizar el conocimiento
}

\author{
Practical inter-learning based on methods and skills to \\ optimize knowledge
}

interlearning prática base em métodos de aprendizagem compartilhada e habilidades para otimizar o conhecimento

\author{
Douglas V. Vásquez-Aguilar \\ douglas.vasqueza@ug.edu.ec \\ ab.vasquezaguilar_10@hotmail.com \\ Luís J. Dominguez de la Torre " \\ luis.dominguezt@ug.edu.ec \\ ljdominguezd@hotmail.com \\ Raúl G. Mata-Muñoz "' \\ raul.matam@ug.edu.ec \\ enginersraulmata@hotmail.com
}

Correspondencia: ab.vasquezaguilar_10@hotmail.com

\footnotetext{
Magister en Diseño Curricular ; Diploma Superior en Pedagogía Universitaria; Licenciado en Ciencias Sociales y Políticas; Abogado de los Tribunales y Juzgados de la República del Ecuador, Universidad de Guayaquil, Ecuador.

Magister en Diseño Curricular; Ingeniero Industrial; Universidad de Guayaquil, Ecuador.

III. Magister en Diseño Curricular; Diploma Superior en Pedagogía Universitaria; Ingeniero Industrial, Universidad de Guayaquil. Ecuador.
} 


\section{Resumen}

El propósito de este estudio basado métodos y competencias fue optimizar el rendimiento académico los estudiantes de la asignatura Relaciones Laborales del 4to año sección nocturna Carrera Ingeniería Industrial Universidad de Guayaquil, en esta investigación cuantitativa participaron 30 estudiantes de la asignatura en mención se realizó una medición en sus trabajos académicos, exposiciones y evaluaciones, el problema se generaba porque el programa analítico estaba obsoleto, antipedagógico y sus contenidos desactualizados, exceso de unidades de análisis, por tal motivo los estudiantes de la asignatura citada no respondían a las expectativas y requerimientos de la sociedad y universidad, además causaba deserción estudiantil, consecuentemente surge la idea de realizar un trabajo de investigación académica basada en métodos y competencias para mejorar el aprendizaje del estudiante nocturno en aula de clases, en esta investigación académica se utilizó el método de encuestas y participaron 30 estudiantes 26 Hombres y 4 Mujeres los mismos que cursaban la asignatura relaciones laborales del cuarto año sección nocturna, el procedimiento utilizado fue el de receptar la evaluación académica escrita de los estudiantes la misma que tuvo una duración de sesenta minutos y sirvió para medir el desempeño y mejorar conocimiento de los aprendientes.

Palabras clave: Métodos; competencia; enseñanza; interaprendizaje; optimizer. 


\begin{abstract}
The purpose of this study based on methods and competencies was to optimize the academic performance of the students of the subject Labor Relations of the 4th year night section Career Industrial Engineering University of Guayaquil, in this quantitative research participated 30 students of the subject mentioned a measurement In its academic works, expositions and evaluations, the problem was generated because the analytical program was obsolete, anti-pedagogical and its contents outdated, excess of units of analysis, for that reason the students of the mentioned subject did not respond to the expectations and requirements of the Society and university, also caused student desertion, consequently arises the idea of performing an academic research work based on methods and skills to improve night student learning in the classroom, in this academic research was used Method of surveys and 30 students participated 26 men and 4 women the same as those who attended the course of work of the fourth year night section, the procedure used was to receive the written academic evaluation of the students the same that lasted sixty minutes and Served to measure performance and improve the knowledge of learners.
\end{abstract}

Key words: Methods; competence; teaching; inter-learning; optimizer. 


\section{Resumo}

O objetivo deste estudo, com base em métodos e competências, foi otimizar o desempenho acadêmico dos alunos da disciplina Relações de Trabalho do $4^{\circ}$ ano noite seção Carreira Engenharia Industrial Universidade de Guayaquil, nesta pesquisa quantitativa participaram 30 alunos do assunto mencionado uma medida Em seus trabalhos acadêmicos, exposições e avaliações, o problema foi gerado porque o programa analítico era obsoleto, anti-pedagógico e seus conteúdos ultrapassados, excesso de unidades de análise, por isso os alunos do referido tema não responderam às expectativas e Requisitos da Sociedade e da universidade, também causou deserção estudantil, consequentemente surge a idéia de realizar um trabalho de pesquisa acadêmica com base em métodos e habilidades para melhorar a aprendizagem do aluno noite em sala de aula, nesta pesquisa acadêmica foi utilizado Método de pesquisas e 30 alunos participaram 26 Homens e 4 mulheres o mesmo que aqueles que assistiram ao curso de trabalho do f $\mathrm{O}$ procedimento utilizado foi o de receber a avaliação acadêmica escrita dos alunos o mesmo que durou sessenta minutos e Servido para medir o desempenho e melhorar o conhecimento dos alunos.

Palavras chave: Métodos; competencia; ensino; inter-aprendizagem; otimizador. 


\section{Introducción.}

Esta investigación académica se produce porque se observó un bajo rendimiento académico de los estudiantes de cuarto año sección nocturna de la asignatura Relaciones Laborales de la Faculta de Ingeniería Industrial Universidad de Guayaquil, el propósito de esta indagación académica fue alcanzar la optimización del conocimiento basado en métodos y competencias de los educandos de la asignatura en mención, , el procedimiento se realizó con la medición en sus trabajos autónomos, académicos, exposiciones y evaluaciones, el problema se generaba por que el programa analítico estaba antiguo, antipedagógico todos su contenidos estaba con muchas unidades analíticas motivo por el cual los aprendientes no respondían a las perspectivas y exigencias de la humanidad y academia asimismo producía deserción estudiantil, razonablemente nace idea de ejecutar un trabajo de búsqueda del conocimiento con el fin mejorar el aprendizaje en educación superior con este trabajo de investigación académica se justifica de manera directa y positiva el perfeccionamiento del aprendizaje en aula de clases, y la aplicabilidad de un plan analítico elaborado con métodos y competencias para la asignatura Legislación Laboral en la carrera de Ingeniería Industrial de la universidad de Guayaquil, en razón que los aprendientes se desenvuelvan con habilidades, competitivos e innovadores de tal manera que brinden sus conocimientos al fortalecimiento de la industrial pública y privada del Ecuador aportando al principio Constitucional del Buen Vivir.

El rendimiento académico, a partir de los resultados de la investigación, especialmente el meta análisis, basado en una gran cantidad de investigaciones y un extenso número de estudiantes. Así, la síntesis meta analítica realizada por (Hattie, 2008) toma en consideración unos 1.000 meta análisis sobre los factores que inciden en el rendimiento académico e incluye alrededor de 60.000 estudios, algunos de los cuales están basados en 5 millones de alumnos y abarcan un total de 245 
millones de estudiantes. Las conclusiones y principios derivados de los resultados de la investigación se pueden articular alrededor de un modelo sobre los factores explicativos del rendimiento académico que incluye: 1) el estudiante, 2) el profesorado, 3) los métodos de enseñanza, 4) la escuela, 5) el contexto familiar y 6) el sistema educativo. Dentro de cada uno de estos factores se han identificado aquellos elementos que muestran un mayor efecto sobre el rendimiento y se divide en ocho temas que recogen los factores anteriores. Cada uno de estos temas se desarrolla como una unidad didáctica. Junto al contenido del mismo se incluyen los objetivos, el resumen, los conceptos clave, las lecturas recomendadas, unas actividades prácticas sugeridas y unos ejercicios de autoevaluación resueltos que pueden servir al estudiante para comprobar los conocimientos adquiridos. El conocimiento de los factores que inciden sobre el rendimiento puede ser de interés para el profesorado, los orientadores y los responsables de la política educativa, para poner en marcha políticas y prácticas basadas en la evidencia de una gran cantidad de resultados obtenidos a nivel internacional y servir de guía para el diseño y desarrollo de nuevas prácticas dirigidas a mejorar la calidad de la educación. (Costa Castejon, 2015)

Las causas y consecuencias del bajo rendimiento académico es un problema que afecta a la mayoría de estudiantes del bachillerato general de las secciones 1, 2, 3, 4, 5 y 6. El rendimiento académico es la evaluación del conocimiento adquirido en el ámbito escolar. En otras palabras es una medida de capacidades del alumno que expresa lo que este ha aprendido a lo largo del proceso formativo. Por lo general los problemas del rendimiento académico suelen aparecer cuando los estudiantes están en la etapa evolutiva de la adolescencia., durante este periodo los cambios que presentan en el adolescente son de origen fisiológico y psicológico, provocan desorientación, angustia e inseguridad para alcanzar metas académicas y personales. Los docentes deberían buenos métodos que incentiven a los alumnos a demostrar el interés por aprender y esto hace que su 
rendimiento sea favorable. Hay que motivar a los alumnos para que aprendan a investigar y a producir conocimientos; de esta manera mejoraran su rendimiento académico. Si se incentivan a pensar, a resolver problemas y aceptar condiciones de trabajo del aula, se puede logar una educación integral con buenos resultados. Debemos buscar donde está la causa del fracaso de los estudiantes si es el acceso a Internet y la inmediatez de la comunicación lo placentero de la actividad de navegar durante horas, chatear, hablar por teléfono o bajar sus tareas de una página web, entre otros factores que causan el bajo rendimiento académico. También otros factores que pueden inciden en los resultados del bajo rendimiento son el entorno del hogar, el nivel de educación de 10os padres, el entorno socioeconómico, los recursos escolares o la sociedad. (Amaya Colocho, 2010)

Basado en la teoría social cognitiva, el objetivo de este trabajo consiste en identificar si existen diferencias de género en el rendimiento académico y en el auto concepto de una muestra de estudiantes de Educación Secundaria Obligatoria de España. Asimismo, se analiza si el auto concepto actúa como predictor del rendimiento académico. Para ello, participaron en el estudio 406 estudiantes (190 alumnas y 215 alumnos) y el cuestionario utilizado para medir el auto concepto fue el AF5. Los resultados muestran que existen diferencias de género a favor de las chicas en las asignaturas: Lengua Española y Literatura. Se encontró también una diferencia marginal en favor de las chicas en la asignatura Idioma extranjero. Además, los estudiantes poseen un auto concepto positivo no sólo en general sino también en las facetas académica, social, emocional, familiar y física. No se aprecian diferencias de género en las anteriores dimensiones. Para terminar, mediante una path análisis se demostró que tanto el auto concepto -académico, familiar y físico- como la edad del estudiante influyen directamente en el rendimiento académico. El estudio también mostró la 
importancia del auto concepto familiar y físico para determinar el auto concepto académico. (Costa \& Tabernero, 2012)

Tras una revisión del marco en que se mueve en la actualidad la Evaluación de Competencias, se enuncian una serie de principios importantes a la hora de elaborar un sistema global. En un segundo momento se hace un planteamiento, partiendo de la modalidad de integración de las competencias genéricas en el desarrollo curricular. Es un planteamiento que se considera en la Universidad de Deusto preferible a otros enfoques, ya que se garantizará el hecho de que el estudiante incorporará, a lo largo de la carrera, comportamientos desempeñados a lo largo de un semestre o de un curso en actividades concretas de las asignaturas. Es más fácil la adquisición de hábitos y, por tanto, el aprendizaje de competencias. En último lugar se presenta una técnica de evaluación de competencias, como es la Entrevista de Evaluación del Aprendizaje Focalizada en Competencias. Se considera, junto a otras técnicas, muy válida para evaluar las competencias genéricas, vinculando la evaluación al desarrollo del aprendizaje. Se fundamenta en la interacción dialógica profesor y estudiante para el desarrollo personal y profesional del alumno. (Sanchez Villa, 2011)

El fortalecimiento, capacitación y actualización de los Docentes de pregrado y postgrado en la facultad de ingeniería industrial es de suma importancia para que el estudiante fortalezca sus conocimientos de forma competitiva para que pueda solucionar los problemas sociales, laborales, que se presenten en las industrias demostrando que en la carrera de ingeniería industrial se busca permanentemente la excelencia académica generando debates, exposiciones, diarias, interactuando y retroalimentándose en el aula de clases con el objetivo de mejorar el proceso de enseñanza y aprendizaje que será desarrollador de manera bilateral y estará orientado a conseguir una excelente 
formación de profesionales competitivos en el campo de la Ingeniería Industrial, en consecuencia las unidades académicas de la Universidad de Guayaquil tendrá que renovar continuamente su programa analítico basado en métodos y competencias por lo menos cada 2 años, con la finalidad de determinar nuevos procedimientos que serán holísticos o integradores, educativos, utilizando diferentes herramientas que permitan mejorar la investigación, para que el estudiante pueda obtener como resultado un mejor proceso de Ínter aprendizaje promoviendo la innovación académica de acuerdo al requerimiento del nuevo modelo de Educación Superior en el Ecuador (El enfoque de competencias CBET, Competencias Based Educación and Training — ha tenido un cierto éxito en la reforma de la FP en algunos países anglosajones. El intento de introducirlo en la Universidad española ha tropezado en el primer paso, la formulación de los objetivos de los títulos en forma de competencias. Ello podría deberse tanto a problemas de comprensibilidad del enfoque de competencias a déficits en la comprensión de las funciones de las Universidades. (Morales Carabaña, 2012)

El presente estudio de investigación académica es de suma importancia porque propone el desarrollo cognitivo en la formación de nuestros estudiantes, construyendo en cada uno de ellos metodologías, competencias y mentalidad de innovadores, emprendedores, para que no solo tenga la visión, de vivir de un puesto de trabajo, sino que, se desempeñe con eficiencia y eficacia para que logre ser efectivo en el cumplimiento de sus objetivos, creando su propia empresa para ofrecer oportunidades de trabajo aportando a la solución de los problemas laboral en el País

El presente estudio tiene como objetivo formar excelente Ingenieros Industriales con conocimientos en métodos y competencias laborales para que aporten con sus sabidurías en las industrias públicas y privadas del Ecuador. 


\section{Materiales y métodos.}

En el presente estudio participaron 30 estudiantes 26 Hombres y 4 Mujeres los mismos que cursaron la asignatura relaciones laborales del cuarto año sección nocturna de la carrera Ingeniería Industrial Universidad de Guayaquil

\section{Tareas y materiales}

En esta tarea instruccional se realizó en la Facultad Ingeniería Industrial Universidad de Guayaquil y consistió en revisar la evaluación cuantitativa de tareas académicas de estudiantes del cuarto año sección nocturna asignatura relaciones laborales

\section{Procedimiento}

El procedimiento seguido en este trabajo de investigación fue el de receptar la evaluación académica escrita de los estudiantes de la asignatura relaciones laborales la misma que tuvo una duración de sesenta minutos y sirvió para medir el desempeño y conocimiento de los mismos.

Análisis de datos

En el presente análisis de datos se utilizó la prueba evaluación de conocimientos de entrada y salida con un nivel significativo.

\begin{tabular}{|l|c|c|c|c|c|}
\hline Pruebas & $\begin{array}{c}\text { Sujetos } \\
\text { Estudiantes }\end{array}$ & Media & $\begin{array}{c}\text { Desviación } \\
\text { estándar }\end{array}$ & $\begin{array}{c}\text { Calificación } \\
\text { más alta }\end{array}$ & $\begin{array}{c}\text { Calificación } \\
\text { más baja }\end{array}$ \\
\hline Entrada & 30 & 5,20 & 1,03 & 7 & 4 \\
\hline Salida & 30 & 8,33 & 0,68 & 9 & 7 \\
\hline
\end{tabular}




\section{Discusión Bibliográfica.}

Julio Carabaña Morales Universidad Complutense de Madrid, competencias y universidad, o un desajuste por mutua ignorancia, Ello podría deberse tanto a problemas de comprensibilidad del enfoque de competencias a déficits en la comprensión de las funciones de las Universidades. (Morales Carabaña, 2012)

Aurelio villa Sánchez Manuel Poblete Ruiz Universidad de Deusto, evaluación de competencias genéricas: principios, oportunidades y limitaciones, El aprendizaje basado en competencias $(\mathrm{ABC})$ es uno de los pilares del cambio. El otro pilar es el sistema europeo de créditos (ECTS). El enfoque de enseñanza-aprendizaje por competencias. (Sanchez Villa, 2011)

Manuel Humberto Ayala Palomino, Desarrollo competencias comunicativas en la enseñanzaaprendizaje El acto de comunicación es un elemento fundamental para la transmisión y generación de conocimientos en los procesos de enseñanza-aprendizaje, tanto docentes como alumnos. (Méndez Morales, Urrea Agraz, \& Ayala Palomino, 2016)

Juan Luis Castejón Costa, El rendimiento académico, a partir de los resultados de la investigación, especialmente el meta análisis, basado en una gran cantidad de investigaciones y un extenso número de estudiantes. (Costa Castejon, 2015)

Colocho Amaya Ligia Sarai, Martínez Platero Claudia Patricia, Membreño Rodríguez Sandra Yamileth, causas y consecuencias del bajo rendimiento académico, Qué duda cabe, que el bajo rendimiento escolar constituye un problema serio y es muy normal que nos preocupe a todos, sobre todo a padres y a profesores. (Amaya Colocho, 2010) 
Sandra Costa y Carmen Tabernero, rendimiento académico y auto concepto en estudiantes, Basado en la teoría social cognitiva, el objetivo de este trabajo consisten en identificar si existen diferencias de género en el rendimiento académico y en el auto concepto de una muestra de estudiantes. (Costa \& Tabernero, 2012)

Guiselle María Garbanzo Vargas, posibles factores asociados al rendimiento académico en estudiantes universitarios, y su vinculación con la calidad de la educación superior pública en general, para lo que se apoya en estudios realizados en España, Colombia, Cuba, México y Costa Rica. Se agrupan en tres categorías: determinantes personales, determinantes sociales y determinantes institucionales que, a su vez, poseen sus propios indicadores. Se hace énfasis en que la búsqueda de la calidad educativa en el sector universitario. (Garbanzo Vargas, 2007)

Adel Alfonso Mendoza, propuesta para la medición del rendimiento académico de los estudiantes de las universidades, El rendimiento estudiantil es un concepto complejo, que aborda distintos aspectos, tanto individuales como colectivos, lo cual condiciona el aprendizaje y la acción académica. En este sentido aborda la actuación de los estudiantes, del docente, así como de los órganos directivos, de la infraestructura y cultura organizacional, por lo cual el rendimiento estudiantil no solo es una calificación y viene concatenada en una política de admisión. (Mendoza, 2011)

Rubén Edel Navarro, el rendimiento académico: concepto, investigación y desarrollo, en la vida académica, habilidad y esfuerzo no son sinónimos; el esfuerzo no garantiza un éxito, y la habilidad empieza a cobrar mayor importancia. esto se debe a cierta capacidad cognitiva que le permite al alumno hacer una elaboración mental de las implicaciones causales que tiene el manejo de las autopercepciones de habilidad y esfuerzo, dichas autopercepciones, si bien son 
complementarias, no presentan el mismo peso para el estudiante; de acuerdo con el modelo, percibirse como hábil es el elemento central. (Navarro, 2013)

Solano, Lester Bernardo, relación entre estilos de aprendizaje y el rendimiento académico en estudiantes, la educación es una tarea muy difícil y requiere de habilidades y destrezas, puesto que el docente debe conseguir que los estudiantes aprendan. Sin embargo los resultados no siempre responden a las expectativas y esfuerzos. Es que el aprendizaje depende de factores diversos: capacidad, motivación, conocimientos previos y estrategias de aprendizaje. (Núñez, Solano, González-Pienda, \& Rosário, 2007)

Fabián Patricio Cordero Méndez, Factores que influyen en el rendimiento académico de los estudiantes de las diferentes facultades ofertadas por la Universidad de Cuenca, objetivo analizar cuáles son los factores determinantes del rendimiento académico de los estudiantes de la universidad de cuenca correspondiente al año 2015 para alcanzar este objetivo se estimó una función de producción educativa mediante el método de Mínimos Cuadrados Ordinarios, en donde se considera como variable dependiente el rendimiento académico del estudiante en función de la edad, nota de la prueba. (Méndez Morales, Urrea Agraz, \& Ayala Palomino, 2016)

\section{Conclusión.}

Esta investigación comprobó que la hipótesis planteada en este trabajo académico es muy positiva con la elaboración y aplicabilidad de un plan analítico basado en métodos y competencias mejoro el desarrollo del conocimiento de estudiantes en la asignatura legislación laboral del cuarto nivel sección nocturna carrera ingeniería Industrial Universidad de Guayaquil 
Douglas V. Vásquez-Aguilar; Luís J. Dominguez de la Torre; Raúl G. Mata-Muñoz

\section{Bibliografía.}

Amaya Colocho, L. (2010). Causas y Consecuencia Bajo Rendimiento Academico. El Salvador: Instituto

General Francisco Menéndez.

Costa Castejon, J. (2015). Aprendizaje y Rendimiento Academico. Cottolengo: Club Universitario.

Costa, S., \& Tabernero, C. (2012). Rendimiento academico y Auto Concepto. Revista Iberoamericana de Psicología y Salud, 3(2), 175-193.

Garbanzo Vargas, G. (2007). Factores asociados al rendimiento académico en estudiantes universitarios, una reflexión desde la calidad de la educación superior pública. Educación, 31(1), 43-63.

Hattie, J. (2008). Visible Learning: A Synthesis of Over 800 Meta-Analyses Relating to Achievement (1 ed.). New York: Routledge.

Méndez Morales, S., Urrea Agraz, G., \& Ayala Palomino, M. (2016). Estrategias de enseñanza-aprendizaje para desarrollar competencias verbal y escrita en un aula de primaria multigrado. Revista de Investigación Educativa de la Escuela de Graduados en Educación, 6(12), 31-36.

Mendoza, A. A. (2011). Propuesta para la medición del rendimiento académico de los estudiantes de la universidad libre utilizando análisis envolvente de datos (DEA). Revista ACADEMIA LIBRE, 8(9), 77-82.

Morales Carabaña, J. (2012). Competencias Universitarias. Madrid: Universidad Complutense de Madrid.

Navarro, R. E. (2013). El rendimiento Académico. Revista Electrónica Iberoamericana sobre Calidad, Eficacia y Cambio en Educación, 1(2), 1-15.

Núñez, J., Solano, P., González-Pienda, J., \& Rosário, P. (2007). El aprendizaje autorregulado como medio y meta de la educación. Papeles del Psicólogo, 27(3), 139-146.

Sanchez Villa, A. (2011). Evaluacion de Competencias Genericas. Bilbao: Universidad de Deusto. 